\title{
OPEN
}
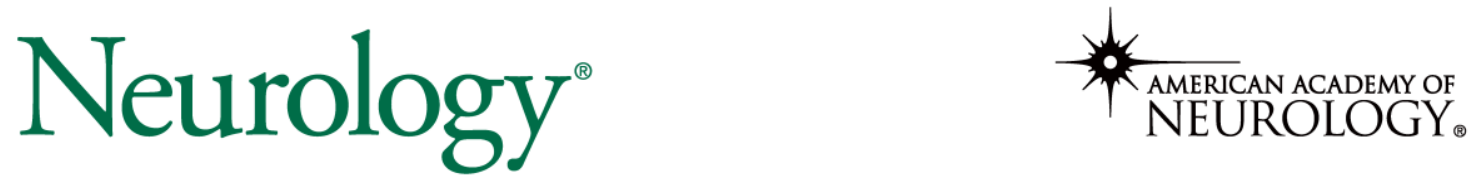

The most widely read and highly cited peer-reviewed neurology journal The Official Journal of the American Academy of Neurology

Neurology Publish Ahead of Print

DOI: 10.1212/WNL.0000000000200017

\section{Cortical Proteins and Individual Differences in Cognitive Resilience in Older Adults}

$\operatorname{Author}(\mathbf{s})$ :

Andrea R Zammit, PhD, MSc ${ }^{1,2}$; Yu LEI, PhD ${ }^{1,3}$; Vladislav Petyuk, $\mathrm{PhD}^{4}$; Julie A. Schneider, $\mathrm{PhD}^{1,3,5}$; Philip Lawrence De Jager, PhD ${ }^{6}$; Hans-Ulrich Klein, $\mathrm{PhD}^{6}$; David A. Bennett, MD ${ }^{1,3}$; Aron S. Buchman, MD ${ }^{1,3}$

\section{Corresponding Author:}

Andrea R Zammit andrea_r_zammit@rush.edu

This is an open access article distributed under the terms of the Creative Commons Attribution-NonCommercial-NoDerivatives License 4.0 (CC BY-NC-ND), which permits downloading and sharing the work provided it is properly cited. The work cannot be changed in any way or used commercially without permission from the journal.

Neurology® Published Ahead of Print articles have been peer reviewed and accepted for publication. This manuscript will be published in its final form after copyediting, page composition, and review of proofs. Errors that could affect the content may be corrected during these processes. 
Affiliation Information for All Authors: 1 Rush Alzheimer' s Disease Center, Rush University Medical Center, Chicago, IL, USA 2 Department of Psychiatry and Behavioral Sciences, Rush University Medical Center, Chicago, IL, USA3 Department of Neurological Sciences, Rush University Medical Center, Chicago, IL, USA 4 Biological Sciences Division, Pacific Northwest National Laboratory, Richland, Washington, United States of America 5 Department of Pathology, Rush University Medical Center, Chicago, IL, USA 6 Center for Translational \& Computational Neuroimmunology, Department of Neurology, Columbia University Medical Center, New York, NY, USA

\section{Contributions:}

Andrea R Zammit: Drafting/revision of the manuscript for content, including medical writing for content; Study concept or design; Analysis or interpretation of data

Yu LEI: Drafting/revision of the manuscript for content, including medical writing for content; Analysis or interpretation of data

Vladislav Petyuk: Drafting/revision of the manuscript for content, including medical writing for content Julie A. Schneider: Drafting/revision of the manuscript for content, including medical writing for content

Philip Lawrence De Jager: Drafting/revision of the manuscript for content, including medical writing for content

Hans-Ulrich Klein: Drafting/revision of the manuscript for content, including medical writing for content

David A. Bennett: Drafting/revision of the manuscript for content, including medical writing for content; Major role in the acquisition of data; Study concept or design; Analysis or interpretation of data Aron S. Buchman: Drafting/revision of the manuscript for content, including medical writing for content; Major role in the acquisition of data; Study concept or design; Analysis or interpretation of data

Figures: 6

Tables: 1

Search Terms: [ 26 ] Alzheimer's disease, [ 36 ] Cognitive aging, [ 54 ] Cohort studies

Acknowledgements: We are deeply indebted to all participants who contributed their data and biospecimens. We are thankful to the staff in the Rush Alzheimer's Disease Center. Data used in this study are available through request via the RADC Research Resource Sharing Hub (https://www.radc.rush.edu/).

Study Funding: This work was supported by National Institute of Health P30AG10161, K01AG054700, R01AG15819, R01AG17917, R01AG47976, R01AG56352, R01AG59732; U01AG46152, and U01AG61356; the Illinois Department of Public Health; and the Robert C. Borwell Endowment Fund. The funding organizations had no role in the design or conduct of the study; collection, management, analysis, or interpretation of the data; or preparation, review, or approval of the manuscript..

Disclosures: A.R. Zammit reports no relevant disclosures relevant to the journal. L. Yu reports no relevant disclosures relevant to the journal. V. Petyuk reports no relevant disclosures relevant to the journal. J.A. Schneider has the following disclosures: Consultant, Alnylam Pharmaceuticals; Consultant, AVID Radiopharmaceuticals (subsidiary or Eli Lilly, Inc); Consultant, Takeda; Expert, National Hockey League. P.L. De Jager reports no relevant disclosures relevant to the journal. H.U. Klein reports no relevant disclosures relevant to the journal. D.A. Bennett reports no relevant disclosures relevant to the journal. A.S. Buchman reports no relevant disclosures relevant to the journal. 


\section{ABSTRACT}

Background/Objective: Cognitive resilience is a well-recognized concept but knowledge gaps about its underlying mechanisms has made it difficult to develop instruments that identify older adults with high or low resilience. We tested if aggregating cortical peptides associated with cognitive resilience into an index can identify adults with higher or lower cognitive resilience.

Methods: We used data from 1,192 older decedents, included annual clinical testing, indices of ten $\mathrm{AD}$ and related dementia (ADRD) pathologies, and 226 proteotypic peptides measured in the dorsal lateral prefrontal cortex. We used linear mixed effects models to identify peptides that were related to cognitive resilience i.e., cognitive decline not explained by ADRD pathologies (FDR <0.05). We aggregated the expression levels of these resilience peptides into a person-specific cognitive resilience index and examined its association with $\mathrm{AD}$ clinical and pathological phenotypes.

Results: We constructed a resilience index from 52 of 226 peptides related to cognitive resilience. A higher index was associated with slower cognitive decline $($ Est. $=0.05$, S.E. $=0.003, p<0.001)$ and slower motor decline $($ Est. $=0.005$, S.E. $=0.001, p<0.001)$. Most resilience peptides $(70 \%)$ were specific to cognitive decline, but $30 \%$ also provided resilience for motor decline. A higher index was also related to a lower burden of $\mathrm{AD}$ pathologies $(\mathrm{OR}=0.41, \mathrm{SE}=0.01, p<0.001)$ and modified the association of AD pathology with cognition, in that a higher index modified the negative effects of $\mathrm{AD}$ pathology on $\mathrm{AD}$ dementia proximate to death $(\mathrm{OR}=0.70, \mathrm{SE}=0.14, p=0.010)$. Up to $90 \%$ of cognitive resilience peptides were related to AD pathological phenotypes.

Discussion: Cortical proteins may provide some degree of cognitive resilience. These multifunctional proteins also seem to provide resilience to other $\mathrm{AD}$ clinical phenotypes and have independent associations with ADRD pathologies. Resilience proteins may be high value therapeutic targets for drug discovery of interventions that maintain brain health in aging adults via multiple pathways. 


\section{Introduction}

Many reports suggest that the heterogeneity of cognitive decline in older adults may be due, in part, to the fact that some individuals are more resilient to ADRD pathologies than others ${ }^{1-4}$. "Cognitive resilience" is often conceptualized as slower than expected cognitive decline despite the presence of brain injury or pathologies ${ }^{5,6}$. It is hypothesized that cognitive resilience emerges from diverse factors including structural, biologic, or behaviors acquired over the life-span that offset the negative effects (i.e., cognitive decline) of accumulating ADRD pathologies ${ }^{5,6}$.

There are several different mechanisms that have been hypothesized to account for cognitive resilience, and which are thought to be dependent on functional and structural brain processes ${ }^{1,7}$. Neurobiological capital, termed "brain reserve", can provide some degree of resilience depending on individual differences in brain structure and anatomy ${ }^{1,7}$. Acquired cognitive skills and abilities termed "cognitive reserve" may also provide resilience to brain aging, insult or pathology, via the efficiency, capacity, and flexibility of one's cognitive systems that help to explain such susceptibility ${ }^{1,8-11}$ Yet, there is a paucity of data about the underlying molecular mechanisms of these distinct but related complex constructs, which has made it difficult to translate these phenotypes into instruments that can be used to identify older adults with high or low cognitive resilience $e^{6,12,13}$.

Attempting to elucidate underlying biological mechanisms that differentiate and identify adults with high or low cognitive resilience is vital to inform on the homogeneity of adults needed in clinical trials for ADRD interventions as well as risk stratification for allocating interventions to vulnerable older adults that might benefit from early treatments. The application of proteomics to investigate cognitive resilience is a novel approach that attempts to explicate the biological mechanisms associated with cognitive resilience. The progression towards a quantifiable measure of cognitive resilience has the potential to advance the field on both theoretical and empirical grounds. 
Resilience proteins may be high value therapeutic targets for drug discovery of personalized resilience therapies that offset the negative cognitive effects of untreatable ADRD pathologies. ${ }^{13}$

Recently, we interrogated a clinical measure of cognitive resilience i.e., cognitive decline not explained by ADRD pathologies, and identified genes ${ }^{14}$ and proteins that may provide cognitive resilience $^{5,6}$. These reports, used either proteome-wide tandem mass tag (TMT) proteomics ${ }^{8}$ or selective reactive monitoring (SRM) targeted proteomics, ${ }^{15}$ to identify a parsimonious group of cognitive resilience proteins. Some cortical resilience proteins were associated with slower cognitive decline, while others were associated with faster cognitive decline. Proteins identified in these discovery studies have been validated in further studies ${ }^{14}$. No study has yet aggregated multiple cortical resilience proteins into a single index that may summarize person-specific indices of resilience.

Building on our prior work ${ }^{5,6,15}$, we used clinical and postmortem data from more than 1,200 older decedents from two independent longitudinal cohorts. We isolated cognitive resilience i.e., cognitive decline unrelated to the negative effects of ADRD pathologies. We then aggregated the expression level of SRM proteins which remained associated with cognitive resilience into a person-specific resilience index. We then tested whether the constructed resilience index could be used to identify individuals with higher or lower than average cognitive resilience.

\section{Methods}

\section{Study participants}

Participants were community-based older adults enrolled in two ongoing cohort studies of aging and dementia, the Religious Orders Study (ROS) ${ }^{16}$ and the Rush Memory and Aging Project $(\mathrm{MAP})^{17}$. Upon enrollment, participants agreed to annual clinical testing and to brain donation after 
death. Uniform annual clinical testing and postmortem assessments are performed in both studies by the same staff facilitating joint analyses. Participants had no known dementia upon enrollment.

\section{Assessment of cognitive function and cognitive status diagnoses}

Detailed annual neuropsychological assessment and clinical examinations were administered $^{18}$. A global composite score was derived by standardizing 17 tests assessing 5 domains of cognitive function using baseline means and standard deviations (SDs) of both cohorts. Repeated cognitive measures were used to estimate the rate of cognitive decline prior to death.

A three-step process based on algorithms and clinical judgment was applied to diagnose Alzheimer's and other dementias, as described previously ${ }^{17,19}$. Final cognitive status was provided by a neurologist at the time of death and after reviewing all clinical data ${ }^{20}$.

\section{Other Clinical Covariates}

Demographic measures, such as age, sex, and number of years in formal education are recorded at enrollment, and age of death is calculated using date of death and date of birth ${ }^{21}$. A global motor score summarized ten common performances, as described previously ${ }^{22}$. Parkinsonism was quantified using the composite measure of 26-items from a modified version of the motor portion of the United Parkinson's Disease Rating Scale (mUPDRS) ${ }^{22}$.

\section{Assessment of ADRD pathologies}

Upon death, the brain was removed and hemisected following standard procedure ${ }^{23}$. One hemisphere was prepared for histological evaluation and the other hemisphere was frozen. The fresh slabs were fixed in 4\% paraformaldehyde. The hemisphere was cut into $1 \mathrm{~cm}$ coronal slabs. Tissue 
blocks from predetermined regions were dissected, embedded in paraffin, and cut into 6 and 20 micro sections ${ }^{23}$. Measured indices included i) AD pathology, ii) hippocampal sclerosis, iii) TDP-43, iv) Nigral neuronal loss, v) Lewy bodies, vi) macro and vii) micro infarcts, viii) cerebral angiopathy (CAA), ix) atherosclerosis, and x) arteriolosclerosis ${ }^{24}$. Neuropathologic data collection and assessment was performed blinded to all clinical and cognitive data, as previously described ${ }^{25,26}$.

\section{Targeted selective reaction monitoring (SRM) proteomics}

Targeted proteomic analysis was performed using frozen tissue from the dorsolateral prefrontal cortex (DLPFC) as part of the Accelerating Medicines Partnership-AD consortium study, following standard protocol ${ }^{5}$. The dorsal lateral prefrontal cortex was chosen based on a large body of work which has identified the prefrontal cortex as a crucial region for cognitive control and for translating ideas or goals into varied behaviors ${ }^{11,27-29}$. It is a region well developed in humans who get $\mathrm{AD}$, and can be devoid of devoid of any brain pathology as opposed to mesial temporal regions which is typically riddled with two or three pathologies with virtually no cases without any pathology ${ }^{30,31}$.A total of 126 genes and a corresponding 226 proteotypic peptides were nominated based on prior literature and research suggesting an association with AD/ADRD clinical and pathological phenotypes. More detailed information can be found in eAppendix1.

\section{Statistical Analysis}

Cognitive resilience proteins. First, we ran growth linear mixed-effects (LME) models allowing random effects on both the intercept and the slope, and adjusting for age at death, sex, and education to investigate person-specific annual rate of cognitive decline (Model 1), with the individuals being the random effect, and the predictors and co-variates being fixed independent variables, and the slope of cognitive decline leading up to death being the outcome. None of the variation around the slope 
was significant in any of the models. Then, we added individual peptides to Model 1 i.e., we ran a total of 226 models (separate models for each peptide) to identify proteotypic peptides associated with cognitive decline (Model 2). Lastly, in each of the 226 models we further adjusted for 10 ADRD pathological indices (Model 3); this enabled us to investigate peptides associated with cognitive resilience i.e., cognitive decline not explained by ADRD pathologies. The latter peptides, associated with cognitive decline after adjustment for ADRD pathologies in Model 3, are termed cognitive resilience proteins. All $p$-values were adjusted using false discover rate $(\operatorname{FDR} p<0.05)$ to correct for multiple testing.

Cognitive resilience index. We multiplied the expression level for each resilience protein by its effect size (i.e. estimate for interaction with annual rate of cognitive decline after controlling for brain pathologies) for each participant. This was repeated for each of the 52 peptides. The 52 scores from each of the peptides were then averaged into a single score for every participant. This was then zscored, for standardization purposes, and termed the cognitive resilience index.

Cognitive resilience index and $A D$ clinical phenotypes. We repeated linear mixed effect models to investigate the person-specific rate of annual cognitive decline as predicted by the constructed cognitive resilience index, adjusting for demographics and ten ADRD pathologies. In a final step, we examined a series of logistic regression models to test whether a higher CRI is related to lower odds of MCI and AD dementia proximate to death. We also employed linear mixed effect models to examine the association of CRI with declining motor function, a non-cognitive ADRD clinical phenotype, using global motor score and parkinsonism prior to death. 
Cognitive resilience index and $A D R D$ pathologies. We used regression models to examine the associations of the constructed cognitive resilience index with ADRD pathologies including the presence of pathologic AD based on NIA Reagan criteria and associations with tau-tangles and beta-amyloid. We added interaction terms to a regression model to examine if the constructed cognitive resilience index modified the known association of AD pathology and cognition. We also examined a series of regression models to determine which of the identified cognitive resilience proteins were related to each of the three AD pathological phenotypes (AD pathology, beta-amyloid, tau). In secondary analyses we also modeled the associations of the cognitive resilience index on non-AD pathological phenotypes. All models were adjusted for age at death, sex, and education. All analyses were carried out using SAS software.

\section{Standard Protocol Approvals, Registrations, and Patient Consents}

Both studies were approved by an Institutional Review Board of Rush University Medical Center. All study participants provided written informed consent as was an Anatomical Gift Act for organ donation.

\section{Data availability}

All data included in these analyses are available the Rush Alzheimer's Disease Center Research Resource Sharing Hub ${ }^{32}$. Descriptions of the studies and data can be found in this hub. Qualified investigators may create an account and submit requests for deidentified data.

\section{Results}

\section{Descriptive characteristics of the study participants}


A total of 1,192 decedents ( $\operatorname{ROS} \mathrm{N}=547,45.9 \%$, MAP $\mathrm{N}=645,54.1 \%$ ), with a mean of $8.7(\mathrm{SD}=4.5)$ years of follow-up, were included in this study. Their clinical characteristics at their last visit proximate to death and postmortem indices are summarized in Table 1.

\section{Identifying cognitive resilience proteins}

We examined 226 separate linear mixed effects models that were adjusted for age at death, sex, and education. Of these, 110 peptides were associated with the rate of cognitive decline (FDR adjusted at $p<0.05)$. Fifty-two of 110 peptides remained associated with cognitive decline, controlling for pathology: Of those, higher levels of 31 peptides and lower levels of 21 peptides were associated with slower cognitive decline, independent of ADRD pathology. Figure 1 illustrates these associations. e'Table 1 lists each of these peptides' estimates, $p$-values and standard errors. e'Table 2 lists each of the peptides' expression means and range of variances.

We reviewed the functions of the 52 identified proteins, and found that the majority of the functions were mitochondrial and synaptic plasticity (e'Table 3, column 2). Next, we examined an exploratory principal components analysis to determine if the 52 proteins might cluster in factors that share common physiologic functions to provide resilience. We retained ten factors with eigenvalues $>1$ and proteins with loadings $>0.50$. Two-thirds of the proteins clustered in the first three factors: The first component, was mainly driven by mitochondrial proteins, the second component consisted of synaptic proteins and the third component was driven by proteins involved in cell structure and function. Full details of this analysis are provided in e'Table 3.

\section{Constructing a Cognitive Resilience Index}

Based on each participant's expression level for each of the 52 proteins that remained associated with cognitive decline after controlling for pathology, we then calculated a person-specific index as described above. As expected, based on its construction, the index was associated with the 
rate of cognitive decline, with a higher resilience index being associated with a slower rate of cognitive decline $($ Est. $=0.05$, S.E. $=0.003, p<0.001)$. The index was not associated with age $(\mathrm{r}=$ 0.03, $\mathrm{p}=0.3)$, or with education $(\mathrm{r}=-0.00006, \mathrm{p}=.998)$. There was no difference between males and females on the index $(\mathrm{t}=-0.05 \mathrm{p}=0.9)$.

To assess whether CRI is independently associated with cognitive decline, we calculated the percentage of variance of cognitive decline accounted for CRI and brain pathologies. First, in separate models relative to terms for demographics alone, CRI accounted for $19.2 \%$ of additional variance of cognitive decline, and brain pathologies accounted for $26.7 \%$ of additional variance of cognitive decline. Then, in the single joint model with both CRI and ADRD pathologies in the model, ARDR pathologies accounted for $22.7 \%$ of variance of cognitive decline, and CRI accounted for $11.8 \%$ of variance. This suggests that the resilience index of aggregated proteins has an independent positive association with cognitive decline that is separate from the well-known negative association of ADRD pathologies with cognitive decline.

We also recalculated the resilience index separately for the two independent samples of older adults analyzed in this study i.e. ROS and MAP. Pearson's correlation for the resilience indices between the two cohorts was .89, indicating the replicability and robustness of the index.

\section{Cognitive Resilience Index and AD clinical phenotypes.}

Cognitive AD phenotypes. In separate regression models, with cognitive status (MCI; AD dementia) as the outcome, a higher CRI was related to lower odds of $\mathrm{MCI}(\mathrm{OR}=0.45,95 \% \mathrm{CI}=0.40-0.51, p$ $<0.001)$ and $\mathrm{AD}$ dementia $(\mathrm{OR}=0.41,95 \% \mathrm{CI}=0.35-0.47, p<0.001)$ proximate to death. Upon further adjusting for pathology, CRI remained associated with lower odds of $\mathrm{MCI}(\mathrm{OR}=0.70$, $95 \% \mathrm{CI}=0.57-0.86, p<0.001)$ and $\mathrm{AD}$ dementia $(\mathrm{OR}=0.66,95 \% \mathrm{CI}=0.51-0.85, p<0.01)$ proximate to death. 
Non-cognitive ADRD phenotypes. To examine the specificity of CRI for cognitive resilience, we repeated the linear mixed-effects model described above replacing the outcome of cognitive decline with global motor decline, and with progressive Parkinsonism [see ${ }^{33}$. CRI was associated with the rate of global motor decline $($ Est. $=0.005$, S.E. $=0.0009, p<0.001)$, and with the rate of progressive Parkinsonism (Est. $=0.005$, S.E. $=0.0009, p<0.001)$.

Figure 2 A, B, and C illustrates model-derived trajectories for rate of cognitive decline (top), motor decline (middle), and for progressive Parkinsonism (bottom), for three representative participants (female, 90 years old, with 15 years of education), with high $\left(90^{\text {th }}\right.$ percentile), average $\left(50^{\text {th }}\right.$ percentile) and low $\left(10^{\text {th }}\right.$ percentile) cognitive resilience indices. The individual with a high resilience index manifests a slower (less steep) trajectory of cognitive decline (black line) compared to an individual with a low resilience score (red line).

Non-cognitive AD phenotypes and individual cognitive resilience proteins. To determine whether any of the 52 peptides associated with cognitive resilience provide resilience for other aging phenotypes implicated in ADRD, we performed a complementary analysis of the associations of the 226 SRM proteins with motor resilience i.e., motor decline manifested by repeated measures of global motor scores not explained by brain pathologies. We found that 14 of the 52 proteins $(26.9 \%)$ used to construct the cognitive resilience index were also associated with motor resilience (e'Table 4).

We repeated a similar analysis examining the associations of these peptides with a second motor phenotype, progressive parkinsonism. We found that 10 of the 52 proteins (19\%), used to construct the index, were also associated with residual progressive parkinsonism i.e., progression not explained by ten brain pathologies (e'Table 4). Combining the results of these three complementary analyses 
shows that most $(\sim 70 \%)$ of the 52 cognitive resilience proteins are specific for cognitive decline and about 30\% may provide resilience for both cognitive and non-cognitive AD phenotypes. (Figure 3;

\section{e'Table 4).}

\section{Cognitive Resilience Index and $\mathrm{AD}$ and non-AD brain pathologies}

ADRD pathologies and cognitive resilience index As shown above, the variance accounted for by CRI with cognitive decline is additive but reduced when combined in the same model with neuropathologies. This raises the question that some of its constituent proteins may also be related to ADRD pathologies. To test this hypothesis, we stratified participants into equal groups of having a high (above median) or a low (below median) cognitive resilience index and examined the distributions and burden of ten ADRD pathologies.

Adults with a high index had fewer ADRD pathologies (mean $=2.7, \mathrm{SD}=1.7$ vs. mean $=$ 3.5, $\mathrm{SD}=1.7)$, and fewer showed a pathologic diagnosis of AD (NIA Reagan) $(35.7 \%$ vs $64.3 \%)$. Frequencies of common ADRD pathologies, stratified by high and low cognitive resilience index, are illustrated in Figure 4. These results that individuals with a higher index had lower ADRD pathologies, and less frequent individuals with a neuropathologic diagnosis of AD suggest that the aggregated peptides may not only provide cognitive resilience, but may also be associated with the measured level of ADRD pathologies.

AD pathological phenotypes and individual peptides. To determine whether any of the 52 peptides associated with cognitive resilience are also related to ADRD pathologies, we used regression analyses to identify which of the 52 cognitive resilience proteins used to construct cognitive resilience index were also associated with AD pathological phenotypes (e'Table 5). Nearly all of the 52 proteins aggregated into the index were also independently associated with AD pathological phenotypes [summary AD pathology score: 47/52 (90\%); tangles: 49/52 (94\%); beta-amyloid: 
$44 / 52(85 \%)]$. Indeed, inspection of the $\mathrm{r}^{2}$ for each of these regression models showed that these proteins accounted for $36 \%, 21 \%$, and $48 \%$ of the variance of these three ADRD pathologies. In Figure 5, we summarize the associations between the cognitive resilience proteins and ADRD pathological phenotypes.

Non-AD pathological phenotypes and the cognitive resilience index. Since ADRD are associated with both AD and non-AD brain pathologies, we used a logistic regression model to examine if the cognitive resilience index was also related to non-AD pathologies. We ran 9 separate models with each neuropathological index as the outcome and CRI as the predictor. A higher index was associated with lower levels of Lewy bodies, nigral neuronal loss, TDP-43, hippocampal sclerosis, and cerebral amyloid angiopathy but not with vascular pathological indices (e'Table 6).

Interaction effects of cognitive resilience index with $A D$ pathological phenotypes. Our prior work suggests that some cortical proteins may modify the known association of AD pathology with cognitive decline ${ }^{34}$. Therefore, we tested if a higher index reduces the strength of the negative association of AD pathology with cognitive decline i.e. is associated with a lower odds of $\mathrm{AD}$ dementia proximate to death. Adding interaction terms between cognitive resilience index and ADRD pathology, we found that $1 \mathrm{SD}$ higher on the index score reduced the detrimental effects of ADRD pathology on dementia proximate to death by $30 \%(\mathrm{OR}=0.70,95 \% \mathrm{CI}=0.53-0.92, \mathrm{SE}=0.14, p=0.01)$, specifically with beta-amyloid $(\mathrm{OR}=0.85,95 \% \mathrm{CI}=0.74-0.98, \mathrm{SE}=0.07, p<0.05)$ but not tangles (e'Table 7, Models A1 and A2). We repeated the analyses using MCI as an outcome, and we found similar results (e'Table 7, Models B1 and B2).

This interaction is illustrated in Figure 6 comparing the odds of dementia for three average participants with high, average and low cognitive resilience index scores. 


\section{Discussion}

We identified and aggregated the expression levels of 52 cognitive resilience proteins measured from postmortem prefrontal cortex, into a single index score, in more than 1,000 wellcharacterized descendants. A higher index was associated with slower cognitive decline, lower probability of MCI and $\mathrm{AD}$ proximate to death, and slower rate of motor decline and progressive parkinsonism. Further analyses showed that some of the cortical proteins used to construct the index provide resilience for cognitive decline as well as resilience for late-life motor decline, and were independently associated with ADRD pathologies. Together, these results suggest that resilience proteins may be high value therapeutic targets for drug discovery of interventions that can offset the negative effects of ADRD pathologies via several different pathways to maintain brain health in aging adults.

While prior work has identified clinical phenotypes for resilience, ranging from behavioral and experiential measures, such as education and engagement in various lifestyle activities 1,8, 9 to molecular mechanisms that include a number of proteins associated with cognitive resilience $5,6,15$, these diverse results have been difficult to operationalize into a tool that might be employed to identify adults with high or low resilience. Risk indices have been employed widely to assess personspecific risk across different diseases, such as coronary heart disease ${ }^{35}$ diabetes $^{36}$, and dementia ${ }^{37}$, and by collecting diverse clinical information (e.g. vascular risk factors and diseases) ${ }^{38}$ and lifestyle measures (e.g. physical activity) ${ }^{39}$, and by aggregating multiple risk factors into an individualized predictive index to identify individuals who may benefit from personalized interventions. To leverage the rapid advances and availability of multilevel streams of genomic data requires new analytic approaches to summarize these large novel datasets into meaningful clinical tools. While our prior work has documented multiple proteins that may provide resilience, we are not aware of 
prior studies that aggregated diverse proteins together into person-specific risk indices of resilience. The risk index constructed in the current study can advance both research and clinical care of older adults. This index is an important first effort in translating genomic data findings into a meaningful clinical measure that provides a framework for elucidating the neurobiology of resilience. This approach would be potentially useful for risk stratification of vulnerable older adults as well as for monitoring the homogeneity of participants in clinical drug trials for ADRD or for assessing responses to interventions targeting neural reserve.

Although we controlled for ADRD pathologies to isolate cognitive resilience, CRI and many of its constituent proteins were also independently related to the level of AD pathology ${ }^{6}$. In prior work we reported that cortical resilience protein expression like small glutamine-rich tetratricopeptide repeat-containing protein beta (SGBT) might also have an independent association with the level of AD pathology. This study lends further support to this idea, as we found that nearly all of the proteins included in the cognitive resilience index were associated with AD pathological phenotypes including tau-tangles, beta-amyloid, and a summary measure of AD pathology. Together these studies highlight that resilience proteins may have multiple functions that contribute to late-life cognitive impairment. Further mechanistic studies are needed to elucidate how resilience proteins drive cognitive decline and ADRD pathologies in aging brains.

While some cortical proteins may be related to the burden of AD pathologies, a prior report in this cohort suggests an additional mechanism, as we found that higher levels of $B D N F$ gene expression may modify the association of a given level of ADRD pathologies (beta amyloid) with the rate of cognitive decline. ${ }^{34}$ The current study extends this prior report as we found that a higher cognitive resilience index also modified the negative effect of ADRD pathologies. Together these analyses provide evidence that cognitive resilience proteins may affect cognitive decline through at least three pathways. First, cognitive resilience proteins may drive cognitive 
decline unrelated to ADRD pathologies. Second, resilience proteins may contribute to mechanisms that affect the level of AD pathology. Third these same proteins may also modify the negative effects of a given level of AD pathology on cognition via an interaction.

Strengths of our study include, leveraging novel resources in a large numbers of older men and women including longitudinal structured clinical assessments using validated instruments, uniform postmortem ADRD pathologies and proteomic data analyzed under a single analytic framework to advance our understanding of the biology underlying cognitive resilience. As with other risk indices, aggregating multiple cortical proteins allowed us to summarize and apply all relevant information using one continuous index. The independent associations of the cognitive resilience index with both cognitive and motor aging phenotypes as well as its validation in independent samples of older adults underscores that the CRI is a robust index which may have clinical utility not only for cognition, but for other important aging phenotypes. Additional analyses highlight that because of the complexity of its constituent proteins, the CRI may have additional functions i.e., it is also related to the burden of ADRD pathologies and may also modify the negative effects of AD pathologic phenotypes.

Limitations: Our results need to be replicated in more diverse populations. While the datasets leveraged in the current study are large, the effects of individual genes and proteins may be quite small and our results highlight the utility of aggregating multiple proteins together into a summary risk score to investigate the biology and heterogeneity of cognitive resilience.

A single brain region was chosen from which to collect the diverse streams of data analyzed in this study, so investigations of other regions of the brain are crucial to identify and quantify the effect of shared and novel region-specific proteins. While ten ADRD pathologies were measured, other pathologies, such as white matter changes ${ }^{40}$ will need to be examined in further studies. This study investigated only a limited number of proteins, so we did not conduct Gene Ontology analysis 
to reduce the number of proteins identified to specific molecular or functional pathways that might facilitate novel treatments. An unbiased proteome-wide study is needed to address this limitation. ${ }^{6}$

The novel correlations reported in this study do not allow for causal inferences and cannot be translated into clinical use without further studies. This study is best conceptualized as an initial step providing support for an approach that can be employed to advance our knowledge about the biology underlying cognitive resilience. Aging brains show combinations of diverse ADRD pathologies so treatments for an individual pathology is likely to have only a small effect on overall cognitive impairment. On the other hand, interventions targeting proteins which drive cognitive resilience could offset the effects of multiple pathologies. Yet, there are thousands of proteins in the DLPFC which may contribute to cognitive resilience. This discovery study, used our novel analytic approach, to identify a parsimonious set of multifunctional cortical proteins which may provide cognitive resilience and resistance to ADRD pathologies. Further experiments in model organisms or human cell modeling will be crucial to characterize the causal mechanisms and pathways which link these resilience proteins with diverse AD clinical phenotypes including cognitive or motor decline as well as pathologic phenotypes like AD. This study also provides investigators novel therapeutic targets for further drug discovery studies to develop treatments that provide resilience even in the presence of untreatable brain pathologies. In the interim, integrative genomic studies may be able to translate our findings from decedents to living adults to catalyze the use of a cognitive resilience index for risk stratification of vulnerable adults in our aging population.

\section{Supplement --- http://links.lww.com/WNL/B808}




\section{References}

1. Stern Y. Cognitive reserve. Neuropsychologia 2009;47:2015-2028.

2. Stern Y. What is cognitive reserve? Theory and research application of the reserve concept. J Int Neuropsychol Soc 2002;8:448-460.

3. Stern Y. Cognitive reserve in ageing and Alzheimer's disease. Lancet Neurol 2012;11:10061012.

4. Katzman R, Terry R, De'Teresa R, et al. Clinical, pathological, and neurochemical changes in dementia: a subgroup with preserved mental status and numerous neocortical plaques. Ann Neurol 1988;23:138-144.

5. Yu L, Petyuk VA, Gaiteri C, et al. Targeted brain proteomics uncover multiple pathways to Alzheimer's dementia. Ann Neurol 2018;84:78-88.

6. Yu L, Tasaki S, Schneider JA, et al. Cortical Proteins Associated With Cognitive Resilience in Community-Dwelling Older Persons. JAMA Psychiatry 2020.

7. Stern Y, Arenaza-Urquijo EM, Bartrés-Faz D, et al. Whitepaper: Defining and investigating cognitive reserve, brain reserve, and brain maintenance. Alzheimer's \& Dementia 2020;16:1305-1311.

8. Boyle PA, Buchman AS, Wilson RS, Yu L, Schneider JA, Bennett DA. Effect of purpose in life on the relation between Alzheimer disease pathologic changes on cognitive function in advanced age. Arch Gen Psychiatry 2012;69:499-505.

9. Scarmeas N, Stern Y. Cognitive Reserve and Lifestyle. Journal of Clinical and Experimental Neuropsychology 2003;25:625-633.

10. Stern Y. The Concept of Cognitive Reserve: A Catalyst for Research. Journal of Clinical and Experimental Neuropsychology 2003;25:589-593.

11. Grafton ST, Volz LJ. From ideas to action: The prefrontal-premotor connections that shape motor behavior. Handb Clin Neurol 2019;163:237-255.

12. Stern Y, Arenaza-Urquijo EM, Bartrés-Faz D, et al. Whitepaper: Defining and investigating cognitive reserve, brain reserve, and brain maintenance. Alzheimer's \& Dementia 2018.

13. Bennett DA. Mixed pathologies and neural reserve: Implications of complexity for Alzheimer disease drug discovery. PLoS Med 2017;14:e1002256.

14. Mostafavi S, Gaiteri C, Sullivan SE, et al. A molecular network of the aging human brain provides insights into the pathology and cognitive decline of Alzheimer's disease. Nat Neurosci 2018;21:811-819.

15. Ramos-Miguel A, Jones AA, Petyuk VA, et al. Proteomic identification of select protein variants of the SNARE interactome associated with cognitive reserve in a large community sample. Acta Neuropathol 2021;141:755-770.

16. Bennett DA, Schneider JA, Arvanitakis Z, Wilson RS. Overview and findings from the religious orders study. Curr Alzheimer Res 2012;9:628-645.

17. Bennett DA, Schneider JA, Buchman AS, Barnes LL, Boyle PA, Wilson RS. Overview and findings from the rush Memory and Aging Project. Current Alzheimer research 2012;9:646663.

18. Wilson RS, Boyle PA, Yu L, Segawa E, Sytsma J, Bennett DA. Conscientiousness, dementia related pathology, and trajectories of cognitive aging. Psychol Aging 2015;30:74-82.

19. Bennett DA, Wilson RS, Schneider JA, et al. Natural history of mild cognitive impairment in older persons. Neurology 2002;59:198-205.

20. Schneider JA, Arvanitakis Z, Bang W, Bennett DA. Mixed brain pathologies account for most dementia cases in community-dwelling older persons. Neurology 2007;69:2197-2204. 
21. Bennett DA, Buchman AS, Boyle PA, Barnes LL, Wilson RS, Schneider JA. Religious Orders Study and Rush Memory and Aging Project. Journal of Alzheimer's disease : JAD 2018;64:S161-S189.

22. Buchman AS, Shulman JM, Nag S, et al. Nigral pathology and parkinsonian signs in elders without Parkinson disease. Ann Neurol 2012;71:258-266.

23. Schneider JA, Aggarwal NT, Barnes L, Boyle P, Bennett DA. The neuropathology of older persons with and without dementia from community versus clinic cohorts. J Alzheimers Dis 2009;18:691-701.

24. Schneider JA, Arvanitakis Z, Yu L, Boyle PA, Leurgans SE, Bennett DA. Cognitive impairment, decline and fluctuations in older community-dwelling subjects with Lewy bodies. Brain 2012;135:3005-3014.

25. Schneider JA, Arvanitakis Z, Leurgans SE, Bennett DA. The neuropathology of probable Alzheimer disease and mild cognitive impairment. Ann Neurol 2009;66:200-208.

26. Boyle PA, Yu L, Wilson RS, Leurgans SE, Schneider JA, Bennett DA. Person-specific contribution of neuropathologies to cognitive loss in old age. Annals of neurology 2018;83:74-83.

27. Miller EK, Cohen JD. An integrative theory of prefrontal cortex function. Annu Rev Neurosci 2001;24:167-202.

28. Miller EK. The prefrontal cortex and cognitive control. Nat Rev Neurosci 2000;1:59-65.

29. Funahashi S, Andreau JM. Prefrontal cortex and neural mechanisms of executive function. J Physiol Paris 2013;107:471-482.

30. Preuss TM, Wise SP. Evolution of prefrontal cortex. Neuropsychopharmacology 2021.

31. Lindberg O, Westman E, Karlsson S, et al. Is the subcallosal medial prefrontal cortex a common site of atrophy in Alzheimer's disease and frontotemporal lobar degeneration? Front Aging Neurosci 2012;4:32.

32. Rush Alzheimer's Disease Center Research Resource Sharing Hub [online]. Available at: https://www.radc.rush.edu. Accessed September, 09, 2021.

33. Buchman AS, Leurgans SE, Boyle PA, Schneider JA, Arnold SE, Bennett DA. Combinations of motor measures more strongly predict adverse health outcomes in old age: the rush memory and aging project, a community-based cohort study. BMC Med 2011;9:42-42.

34. Buchman AS, Yu L, Boyle PA, Schneider JA, De Jager PL, Bennett DA. Higher brain BDNF gene expression is associated with slower cognitive decline in older adults. Neurology 2016;86:735-741.

35. D'Agostino RB, Sr., Grundy S, Sullivan LM, Wilson P. Validation of the Framingham coronary heart disease prediction scores: results of a multiple ethnic groups investigation. Jama 2001;286:180-187.

36. Lindström J, Tuomilehto J. The diabetes risk score: a practical tool to predict type 2 diabetes risk. Diabetes Care 2003;26:725-731.

37. Sindi S, Calov E, Fokkens J, et al. The CAIDE Dementia Risk Score App: The development of an evidence-based mobile application to predict the risk of dementia. Alzheimers Dement (Amst) 2015;1:328-333.

38. Wolf PA, D'Agostino RB, Belanger AJ, Kannel WB. Probability of stroke: a risk profile from the Framingham Study. Stroke 1991;22:312-318.

39. Chiuve SE, Cook NR, Shay CM, et al. Lifestyle-Based Prediction Model for the Prevention of CVD: The Healthy Heart Score. Journal of the American Heart Association;3:e000954.

40. Kim N, Yu L, Dawe R, et al. Microstructural changes in the brain mediate the association of AK4, IGFBP5, HSPB2, and ITPK1 with cognitive decline. Neurobiology of aging 2019;84:17-25. 


\section{Tables}

Table 1. Clinical Measures at Last Visit before Death and Neuropathological Characteristics of the analytic cohort $(\mathrm{N}=1192)$.

\begin{tabular}{|c|c|}
\hline Measure & Mean (SD)/ n (\%) \\
\hline \multicolumn{2}{|l|}{ Clinical measures } \\
\hline Age at baseline, years & $80.5(7.0)$ \\
\hline Age at death, years & $89.3(6.5)$ \\
\hline Sex, Male & $382(32 \%)$ \\
\hline Education, years & $16.2(3.6)$ \\
\hline Race, white & $1105(97.4 \%)$ \\
\hline $\begin{array}{l}\text { Mini-Mental State Examination } \\
(0-30)\end{array}$ & $20.5(9.4)$ \\
\hline Global cognition score & $-0.99(1.2)$ \\
\hline AD Dementia & $516(43.3 \%)$ \\
\hline Mild cognitive impairment & $290(24.3 \%)$ \\
\hline Post mortem indices & 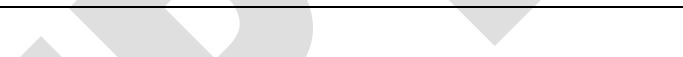 \\
\hline Postmortem interval (hours, SD) & $8.4(6.0)$ \\
\hline $\begin{array}{l}\text { Nigral neuronal loss (moderate- } \\
\text { severe) }\end{array}$ & $155(13.1 \%)$ \\
\hline Lewy bodies (present) & $316(26.6 \%)$ \\
\hline Pathologic AD (NIA Reagan) & $766(64.3 \%)$ \\
\hline $\begin{array}{l}\text { TDP-43 (present beyond } \\
\text { amygdala) }\end{array}$ & $380(32.3 \%)$ \\
\hline Hippocampal Sclerosis (present) & $113(9.5 \%)$ \\
\hline Macroinfarcts present & $430(36.1 \%)$ \\
\hline Microinfarcts present & $337(28.3 \%)$ \\
\hline Atherosclerosis (moderate-severe) & $405(34.1 \%)$ \\
\hline $\begin{array}{l}\text { Arteriolosclerosis (moderate- } \\
\text { severe) }\end{array}$ & $397(33.5 \%)$ \\
\hline $\begin{array}{l}\text { Cerebral amyloid angiopathy } \\
\text { (moderate-severe) }\end{array}$ & $422(36 \%)$ \\
\hline
\end{tabular}




\section{Figures}

Figure 1: Fifty-two peptides are independently associated with cognitive resilience. Individual peptides measured in the dorsal lateral prefrontal cortex are represented by squares. Each row shows the results for the interaction of a single peptide with the annual rate of cognitive decline for an average of about nine years of follow-up. This term derives from a linear mixed-effect model which included 15 terms including time (annual rate of cognitive decline) age, sex, education, expression level of a single peptide and terms for each of the 10 brain pathologies as well as their interaction with time. Squares on the left from 0 represent a negative association with cognitive decline i.e., faster cognitive decline for higher levels of protein, while squares on the right from 0 represent a positive association with cognitive decline i.e., slower cognitive decline for a higher level of protein. Bars, $95 \%$ confidence intervals (CIs). The y-axis on the right displays the standard errors and $p$-values of individual peptides (FDR $<0.05$ ).

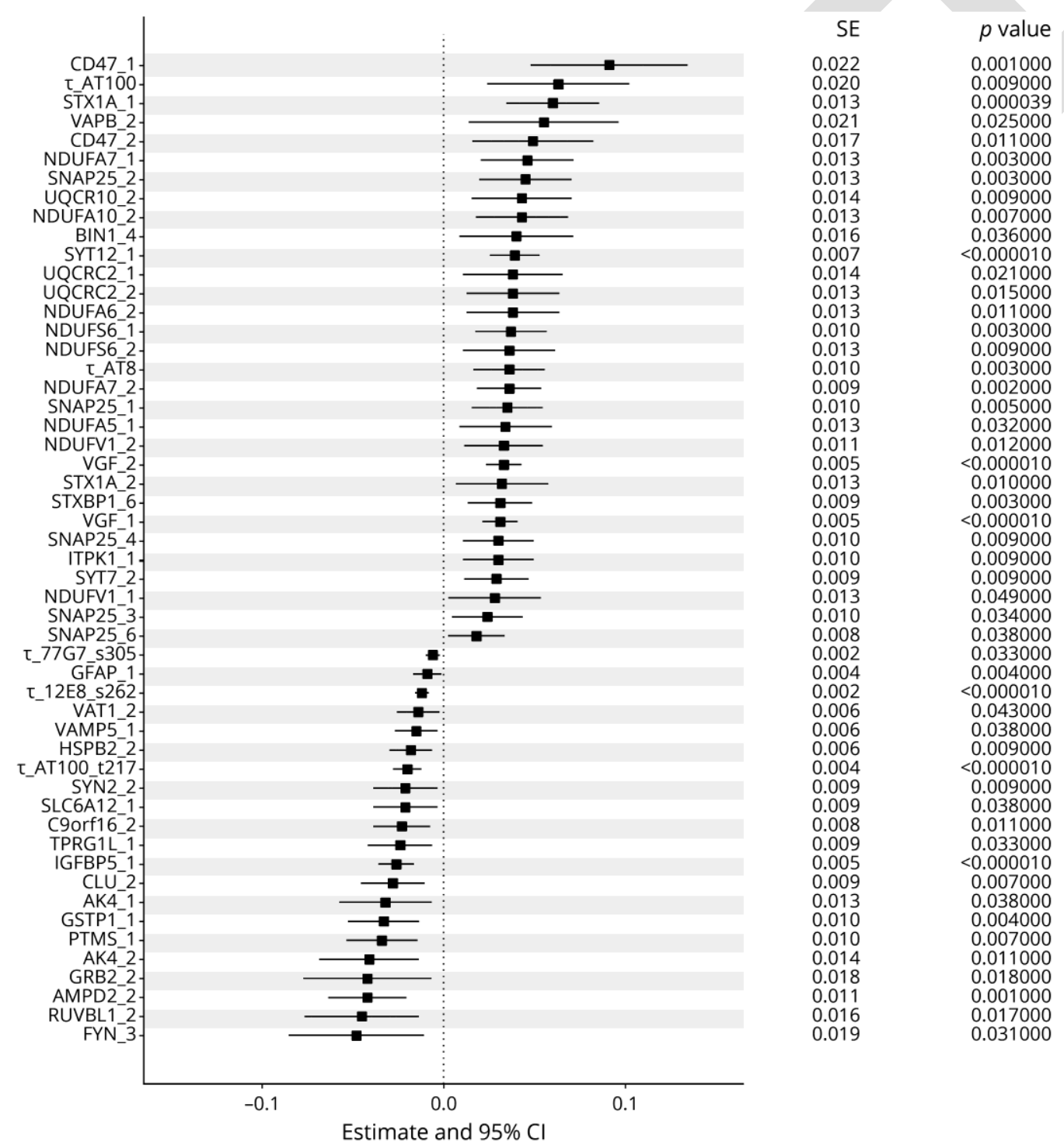


Figure 2. Cognitive resilience index and late-life cognitive and motor impairment. Model derived trajectories for three average participants: 90 -year-old female with 15 years of education contrasting rate of decline for low (10 $0^{\text {th }}$ percentile-red line), average ( $50^{\text {th }}$ percentile blue line) and high $\left(90^{\text {th }}\right.$ percentile-black line) cognitive resilience index. An individual with low vs high CRI showed 2.6-fold faster rate of cognitive decline; 1.33 -fold faster rate of motor decline and 1.31-fold faster rate of progressive parkinsonism.
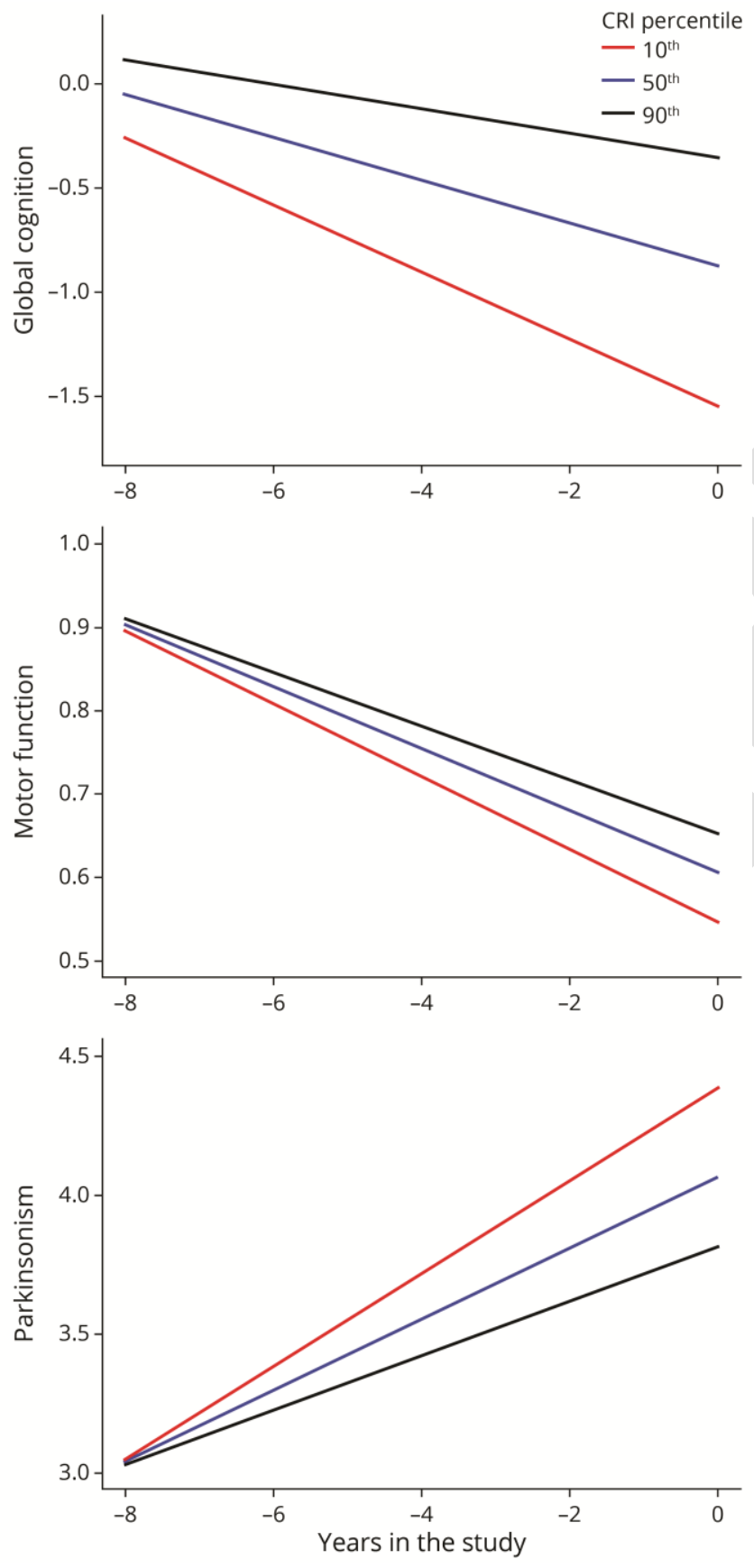
Figure 3. Some cortical proteins may provide resilience for a specific aging phenotype and some provide resilience for more than one phenotype. In this figure, the dashed and dotted lines emerging from the "Prefrontal SRM Proteins box shows the results of three complementary analyses of the number of cortical proteins $(\mathrm{N}=226)$ measured in the dorsolateral prefrontal cortex related to cognitive resilience ( $\mathrm{n}=$ 52), to motor resilience $(\mathrm{n}=20)$, and to Parkinsonism Resilience $(\mathrm{n}=16)$ after controlling for brain pathologies (FDR <0.05). Below the boxes for motor and parkinsonism resilience are the number of proteins shared with cognitive resilience. The Venn diagram shows the number of proteins shared and unique for each of the three phenotypes. About $70 \%$ of the proteins used to construct CRI provide resilience for only cognition and about 30\% may also provide motor resilience. A smaller number of proteins from the prefrontal cortex not included in the CRI may provide resilience for one or more aging motor phenotypes. These latter proteins are located outside the "green" overlapping cognitive and motor areas in the figure.

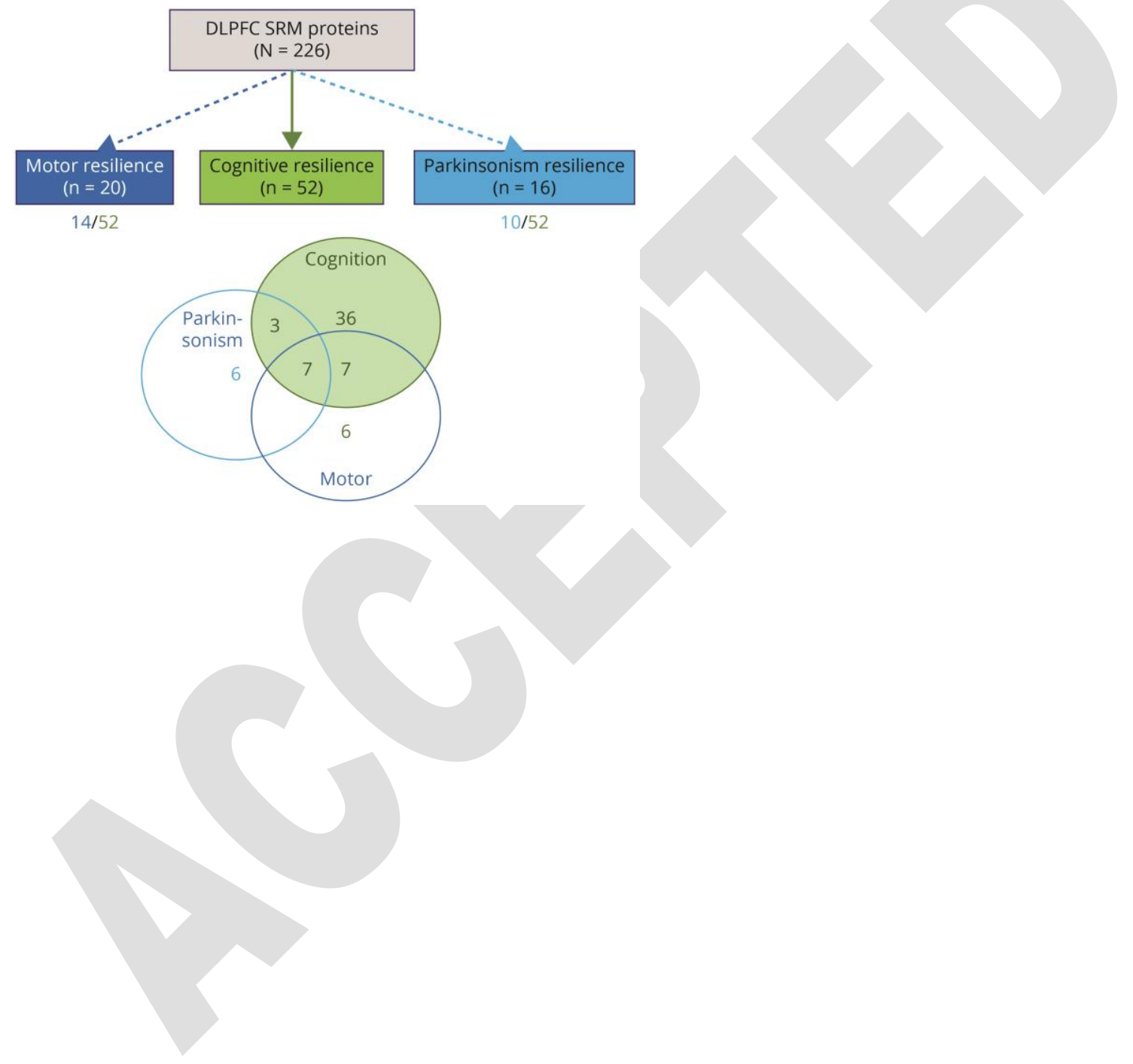


Caption: Figure 4. The burden of brain pathologies is lower in individuals with high CRI scores. The bar chart (B) shows the frequencies of mixed-brain pathologies in the analytic cohort. (C) Connected black dots on the $\mathrm{x}$-axis indicate the specific combination of neuropathologies represented. (A) Histograms in the main panel show the frequencies of the neuropathologic indices for participants with high CRI (>median CRI, light blue) and participants with low CRI ( $<$ median CRI, black). As illustrated in the figure, brain neuropathologic indices, frequently co-occur. Overall, fewer combinations of different brain pathologies are observed in individuals with high CRI.

A

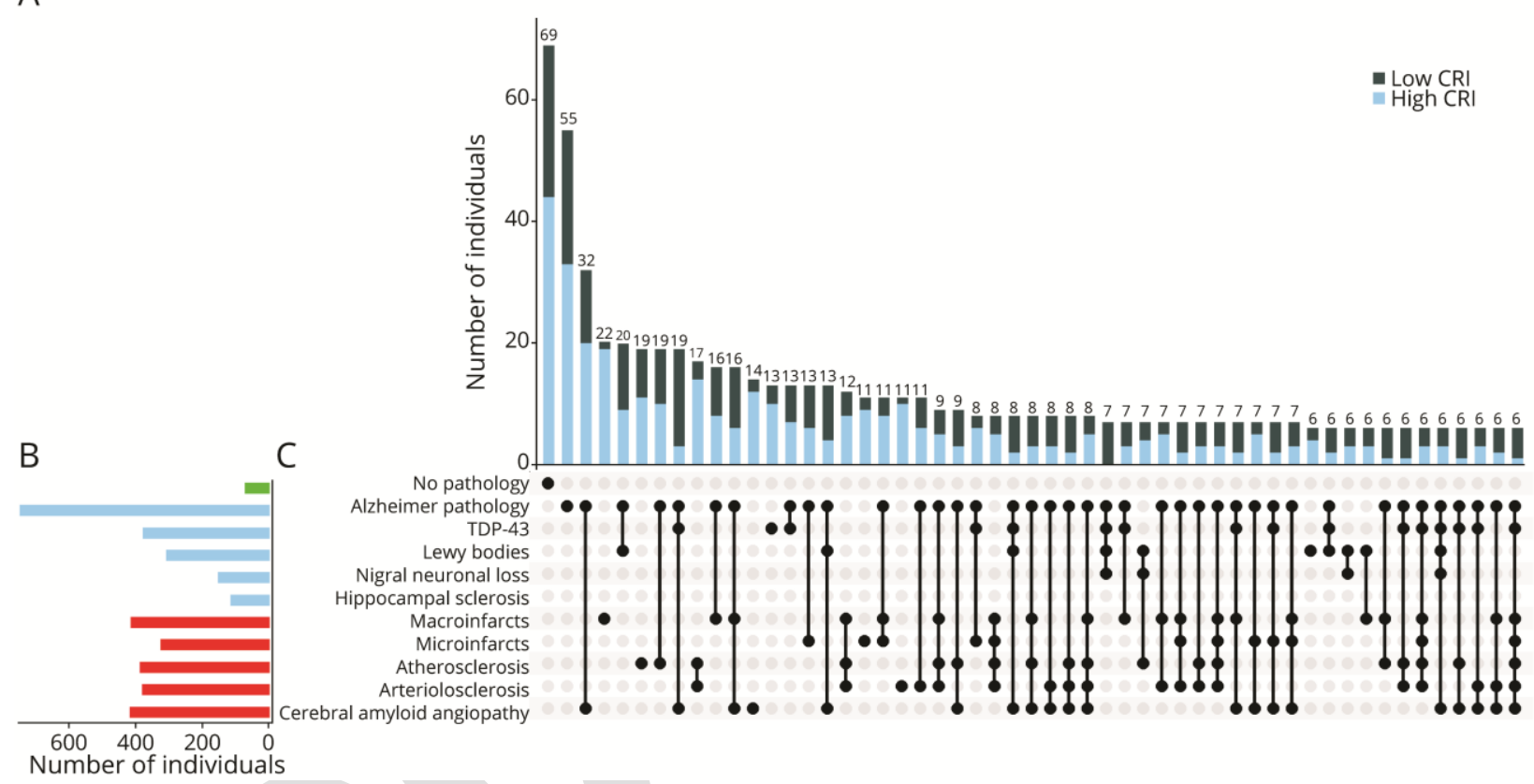


Figure 5. Cortical proteins are multifunctional and may provide resilience for aging phenotypes and are also associated with $\mathrm{AD}$ pathological phenotypes. In this figure in panel $\mathrm{A}$, we illustrate that many of the cortical proteins included in the CRI are also associated with AD pathologic phenotypes including a summary score for AD pathology, beta- amyloid, and tau-tangles. The Venn diagram in panel B, illustrates most of the 52 cognitive resilience proteins are also associated with AD pathological phenotypes.

A

$\mathrm{B}$

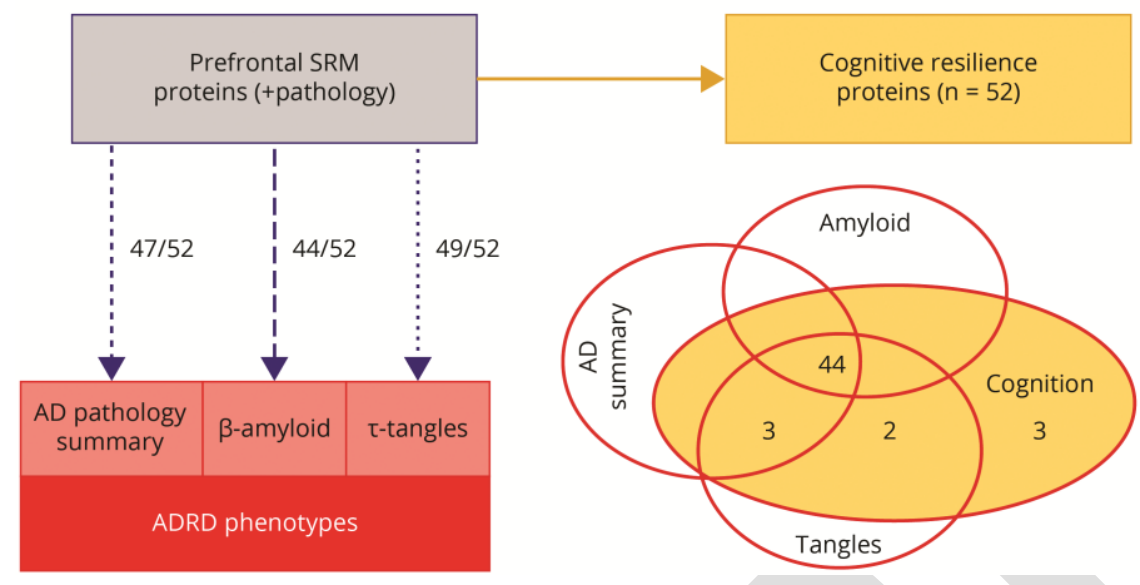

Cognitive resilience proteins $(n=52)$ 
Figure 6. The association of $\mathrm{AD}$ pathology with the probability of dementia proximate to death varies with an individual's level of CRI. Illustration of the probability of clinically diagnosed AD dementia proximate to death as a function of the level of AD pathology (summary measure) in participants with low (in red, $10^{\text {th }}$ percentile), average (in blue, $50^{\text {th }}$ percentile), and high (in black, 90th percentile) cognitive resilience index. For all measures of AD pathology (X axis), an individual with high CRI (black line) has a lower probability of dementia compared to an individual with low CRI (red).

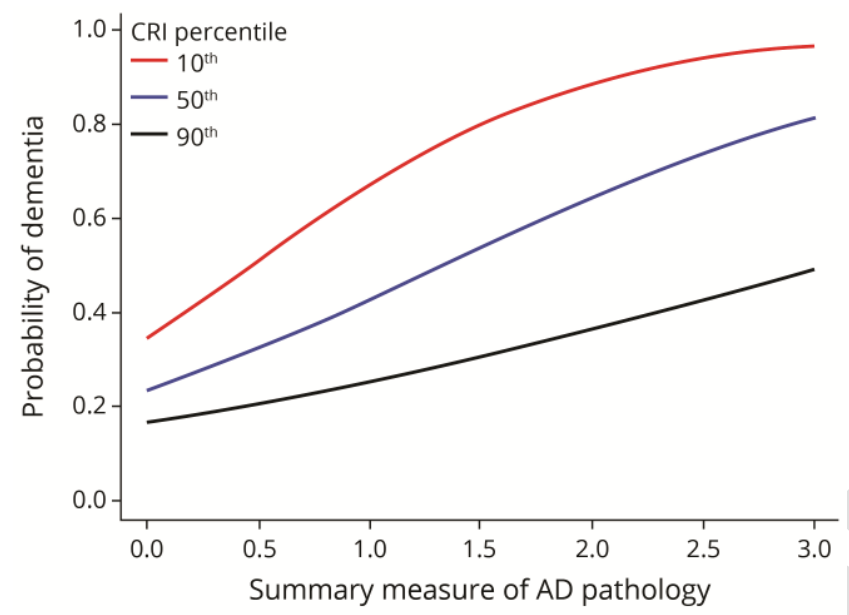




\section{Neurology}

\section{Cortical Proteins and Individual Differences in Cognitive Resilience in Older Adults Andrea R Zammit, Yu LEI, Vladislav Petyuk, et al. \\ Neurology published online March 3, 2022 \\ DOI 10.1212/WNL.0000000000200017}

\section{This information is current as of March 3, 2022}

\section{Updated Information \& Services}

Citations

Subspecialty Collections

Errata

Permissions \& Licensing

Reprints including high resolution figures, can be found at: http://n.neurology.org/content/early/2022/03/03/WNL.0000000000200017.f ull

This article has been cited by 2 HighWire-hosted articles: http://n.neurology.org/content/early/2022/03/03/WNL.0000000000200017.f ull\#\#otherarticles

This article, along with others on similar topics, appears in the following collection(s):

Alzheimer's disease

http://n.neurology.org/cgi/collection/alzheimers_disease

Cognitive aging

http://n.neurology.org/cgi/collection/cognitive_aging

Cohort studies

http://n.neurology.org/cgi/collection/cohort_studies

An erratum has been published regarding this article. Please see next page or:

/content/early/2022/11/29/WNL.0000000000201623.full.pdf

Information about reproducing this article in parts (figures,tables) or in its entirety can be found online at:

http://www.neurology.org/about/about_the_journal\#permissions

Information about ordering reprints can be found online:

http://n.neurology.org/subscribers/advertise

Neurology ${ }^{\circledR}$ is the official journal of the American Academy of Neurology. Published continuously since 1951, it is now a weekly with 48 issues per year. Copyright Copyright $@ 2022$ The Author(s). Published by Wolters

Kluwer Health, Inc. on behalf of the American Academy of Neurology.. All rights reserved. Print ISSN:

0028-3878. Online ISSN: 1526-632X.

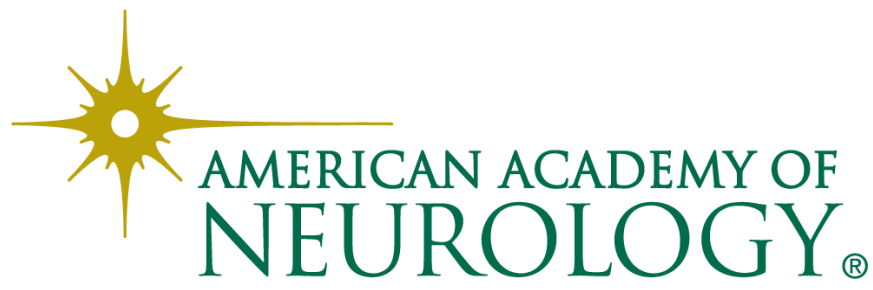




\section{Neurology}

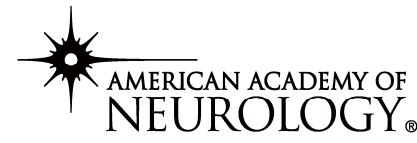

The most widely read and highly cited peer-reviewed neurology journal

The Official Journal of the American Academy of Neurology

\section{Neurology Publish Ahead of Print \\ DOI: 10.1212/WNL.0000000000201623}

\section{CORRECTION}

\section{Cortical Proteins and Individual Differences in Cognitive Resilience in Older Adults}

In the Research Article "Cortical Proteins and Individual Differences in Cognitive Resilience in Older Adults" by Zammit et al.., the row for protein GFAP_1 in eTable 4 should appear in the pink section rather than the orange section. Thus, the number shown in the overlap for Motor and Cognition in the Venn diagram in Figure 3 should be 8; further, the numbers for Motor alone and Cognition alone should be 5 and 34 , respectively. The authors regret the error.

\section{REFERENCE}

${ }^{1}$ Zammit A, Yu L, Petyuk V, et al. Cortical Proteins and Individual Differences in Cognitive Resilience in Older Adults. Neurology 2022;98:e1304-e1214.

Neurology® Published Ahead of Print articles have been peer reviewed and accepted for publication. This manuscript will be published in its final form after copyediting, page composition, and review of proofs. Errors that could affect the content may be corrected during these processes. 